\title{
THE NONVARIABILITY OF THE PROGENITOR OF SUPERNOVA 1993J IN M81
}

\author{
Judith G. COHEN, Jeremy Darling, AND Alain Porter ${ }^{1}$ \\ Palomar Observatory, California Institute of Technology, Mail Code 105-24, Pasadena, California 91125 \\ Electronic mail: jlc@astro.caltech.edu, darling@ugsc.caltech.edu
}

\begin{abstract}
An archive of images taken in the early 1980s exists for a field which includes the progenitor of supernova 1993J in M81. Fifty-four CCD frames in $V$, and several in other colors from this set taken in 1984 are analyzed to demonstrate that the progenitor of supernova 1993J was not variable to within a limit of 0.2 mag. The frames analyzed cover a time span of 150 days. The colors of the M81 SN progenitor are consistent with those of a late G supergiant. (C) 1995 American Astronomical Society.
\end{abstract}

\section{INTRODUCTION}

Supernova 1993J is extremely unusual in several respects. It was discovered on 1993 March 18.906 by Fransicso Garcia (Ripero 1993) in M81, a galaxy which is sufficiently nearby that Cepheids have been observed, both from the ground (Freedman \& Madore 1988) and with HST (Freedman et al. 1994). This of course makes the supernova bright and easy to study in detail at many wavelengths. The supernova itself was peculiar. Its lightcurve at optical wavelengths shows a double peak (Benson et al. 1994; Richmond et al. 1994), and its spectrum evolved from a Type II at early times to a type $\mathrm{Ib}$ at late times (Filippenko et al. 1993; Swartz et al. 1993). This has led to much speculation that SN 1993J occurred in a massive star which had lost nearly all of its hydrogen-rich envelope prior to the explosion, either via mass loss associated with the normal course of stellar evolution or through mass transfer in a binary system (Hoflich et al. 1993; Schmidt et al. 1993; Podsiadlowsky et al. 1993).

Because M81 is so nearby, there exist some observations of the progenitor of this supernova, and it is on this aspect of the event that we focus our attention here. With the exception of SN 1987A in the LMCV (see review by Arnett et al. 1989, and especially Hazen 1987), this is the only case where the progenitor was bright enough to have been observed. Those observations of the progenitor star of SN 1993J available in early 1993 were summarized by Benson et al. (1994). They consisted of a collection of CCD images through random filters taken by random people at random times with various telescopes and instruments covering the period from 1982 to 3 Feb. 1993. In their compilation there are four $B$ detections, four $V$ detections, six $R$ detections, and two $I$ detections of the progenitor star. Detections, as distinct from upper limits, were contributed by their group and by Perelmutter (1993), Humphreys et al. (1993), Magnier et al. (1993), Cohen (1993), Prugniel (1993), Blakeslee \& Tonry (1993), Salzer et al. (1993), and Tomaney \& Crotts (1993).

Benson et al. commented that based on their compilation the progenitor star appeared to be variable. However, Aldering et al. (1994) reviewed these observations, redid the analysis from the original data in several cases, and at-

${ }^{1}$ Deceased. tempted to use common photometric calibrations for all the data. They revised Perelmutter's and Magnier et al.'s magnitudes substantially, producing much better agreement among this heterogeneous sample. They also suggest that several stars (plus perhaps nebular emission) may be contributing to the observed flux of the supernova progenitor at the bluest wavelengths, where the image appears to be slightly extended, but there was no evidence for an extended image at red wavelengths.

As part of a program to detect novae in M81, J. C. routinely monitored several fields in M81 in the early 1980's. The data for 1984 have been analyzed here. We find no evidence for variability of the progenitor of supernova 1993J during the period of observation.

\section{2: ANALYSIS OF THE 1984 DATA}

M81 was observed repeatedly in the early 1980 s with the $1.5 \mathrm{~m}$ telescope on Palomar Mountain in an attempt to detect novae in that galaxy. The observations in 1984 used an RCA $320 \times 512 \mathrm{CCD}$ detector with a Johnson $V$ filter. Occasionally frames through the Thuan \& Gunn (1976) $g$ and $r$ filters were obtained. Exposures were obtained on every assigned night that was not so cloudy that it was hopeless, irrespective of the seeing or of the transparency. All $V$ exposures were 360 s long.

While the quantum efficiency of this detector is reasonably good, especially in the blue, its readout noise is fairly high (30 electrons RMS) compared to the current state of the art devices and it had a very high apparent "cosmic ray" rate, which was probably due to a poor choice of materials by RCA somewhere in the CCD packaging. It is this high "cosmic ray" rate which led in part to the failure of the nova search program.

The scale of the detector at the $1.5 \mathrm{~m}$ telescope using reimaging optics is $1.2 \mathrm{arcsec} / \mathrm{pixel}$. Thus the field is $6 \times 10$ arcmin, and M81 was covered using six separate fields, which had some overlap among them. The location of SN 1993J appears in two of these fields.

The frames of those two sectors of M81 from 1984 were recovered from the old backup tapes and were analyzed. The best 54 frames taken through the $V$ filter were used. (There were a total of about $80 \mathrm{~V}$ frames taken during 1984, but the ones with the worst seeing and/or poor transparency were 
TABLE 1. Derived magnitudes of local standard stars

\begin{tabular}{llrr}
\hline \hline Star ID & V & $\delta$ (East) & \multicolumn{2}{c}{$\begin{array}{r}\text { (North) } \\
\end{array}$} & (mag) & (arc-sec & from SN) \\
\hline Star 2 & 17.63 & 2 & 24 \\
Star 4 & 17.90 & -22 & -55 \\
Star 1 & 18.92 & -25 & 41 \\
Star 3 & 20.09 & 1 & 11 \\
\hline \hline
\end{tabular}

rejected.) There are also two $r$ frames and one $g$ frame. Photometric standards from Thuan and Gunn were observed in 1984 on the same nights as M81 when the weather was suitable.

To analyze the data, the standard aperture photometry code of Figaro was used with a radius of 2 pixels $(2.4$ arcsec). Photometry of four local standard stars selected to be near the progenitor of the supernova, as well as that of the progenitor itself, was obtained from each of the good frames. Three of these local standard stars are quite bright, while the fourth is only slightly brighter than the M81 supernova progenitor. Although de Vaucouleurs et al. (1994) have redone the photometric calibration for 36 comparison stars around SN 1993J, this was intended as an aid to those observing the supernova during its decline. All of their objects are too bright to avoid saturating the CCD images and most are lo- cated outside the rather small field of our CCD images. However, they quote (as a private communication from Humphreys and Prugniel) magnitudes for a star (star A) located 25 arcsec NNE of the SN, $V=17.78 \mathrm{mag}, B-V \approx 1.24 \mathrm{mag}$, $V-R_{C} \approx 0.85 \mathrm{mag}$. This star was one of the three bright local standard stars we adopted.

Using observations of M81 carried out on eight photometric nights during early 1984 and magnitudes measured for large apertures around the images of the Thuan-Gunn standard BD26-2606, we derive the magnitudes for the three local bright standard stars we use that are listed in Table 1.

In the Thuan-Gunn system, we only have data on two photometric nights, and we find for Star 2, $r=17.26 \pm 0.04$ mag and $g=17.91 \pm 0.04 \mathrm{mag}$. Although the published value for $V$ (Star 2=Star A) is 0.15 mag fainter than we obtained, we use our values, fearing contamination from the much brighter supernova in the recent determinations, and hesitating to trust the poorly calibrated and anecdotal published magnitudes; the reader should be aware of this discrepancy.

For the observations through the $V$ filter of the relevant sectors of M81, Table 2 lists the date of the observation, the magnitude of the faintest local standard star, and the magnitude we obtain for the progenitor of SN 1993J in M81 for 54 epochs in 1984. The mean $V$ magnitude of this object is $20.58 \pm 0.17 \mathrm{mag}$ from 51 frames. $\langle V\rangle$ for the faint local standard is $20.09 \pm 0.15 \mathrm{mag}$ from 54 frames. The errors are the $1 \sigma$ deviation about the mean of the data. The formal errors computed by the aperture photometry program are based on the photon statistics of the object and of the sky. They are

TABLE 2. $V$ magnitudes of the progenitor of the M81 supernova.

\begin{tabular}{lll|lll}
\hline \hline $\begin{array}{l}\text { Julian Date } \\
(-2445000)\end{array}$ & $\begin{array}{l}\text { V(Star 3) } \\
(\mathrm{mag})\end{array}$ & $\begin{array}{l}\text { V(SN prog. }) \\
(\mathrm{mag})\end{array}$ & $\begin{array}{l}\text { Julian Date } \\
(-2445000)\end{array}$ & $\begin{array}{l}\text { V(Star 3) } \\
(\mathrm{mag})\end{array}$ & $\begin{array}{l}\text { V(SN Prog. }) \\
(\mathrm{mag})\end{array}$ \\
\hline 708.949 & 20.023 & 20.398 & 798.797 & 20.307 & 20.567 \\
710.847 & 20.035 & 20.325 & 811.652 & 19.843 & 20.600 \\
753.683 & 19.896 & 20.587 & 811.672 & 20.012 & 20.488 \\
754.722 & 20.023 & 20.605 & 811.774 & 20.305 & 20.597 \\
755.700 & 19.991 & 20.892 & 812.651 & 20.212 & 20.499 \\
755.721 & 20.326 & 20.351 & 812.779 & 20.062 & 20.531 \\
755.801 & 19.929 & 20.322 & 813.679 & 20.241 & 20.366 \\
765.640 & 20.069 & 20.814 & 824.661 & 20.268 & 20.575 \\
766.646 & 20.212 & 20.624 & 824.749 & 19.880 & 20.460 \\
766.866 & 20.014 & 20.523 & 824.768 & 20.253 & 20.653 \\
767.763 & 20.441 & 20.810 & 825.662 & 20.178 & 20.502 \\
768.656 & 20.131 & 20.538 & 825.756 & 20.063 & 20.779 \\
769.686 & 20.234 & 20.809 & 825.775 & 20.191 & 20.721 \\
770.839 & 19.934 & 20.309 & 827.757 & 20.272 & 20.401 \\
770.888 & 20.111 & 20.775 & 827.776 & 20.074 & 20.559 \\
770.902 & 20.108 & 20.522 & 828.754 & 20.404 & 20.659 \\
779.640 & 20.017 & 20.372 & 828.773 & 19.989 & 20.383 \\
780.708 & 20.108 & 20.999 & 839.667 & 20.023 & 20.745 \\
782.656 & 20.007 & 20.858 & 840.688 & 19.910 & 20.460 \\
782.691 & 20.057 & 20.840 & 841.667 & 19.931 & 20.503 \\
783.642 & 20.130 & 20.482 & 841.686 & 19.855 & 20.523 \\
797.926 & 20.067 & 20.546 & 842.669 & 20.231 & 20.429 \\
798.649 & 19.980 & 20.905 & 842.688 & 20.331 & 20.560 \\
798.669 & 20.311 & 20.460 & 844.676 & 20.004 & 20.633 \\
798.699 & 20.147 & 20.606 & 844.699 & 19.946 & 20.351 \\
798.704 & 19.972 & 20.562 & & & \\
\hline \hline & & & & & \\
\hline
\end{tabular}




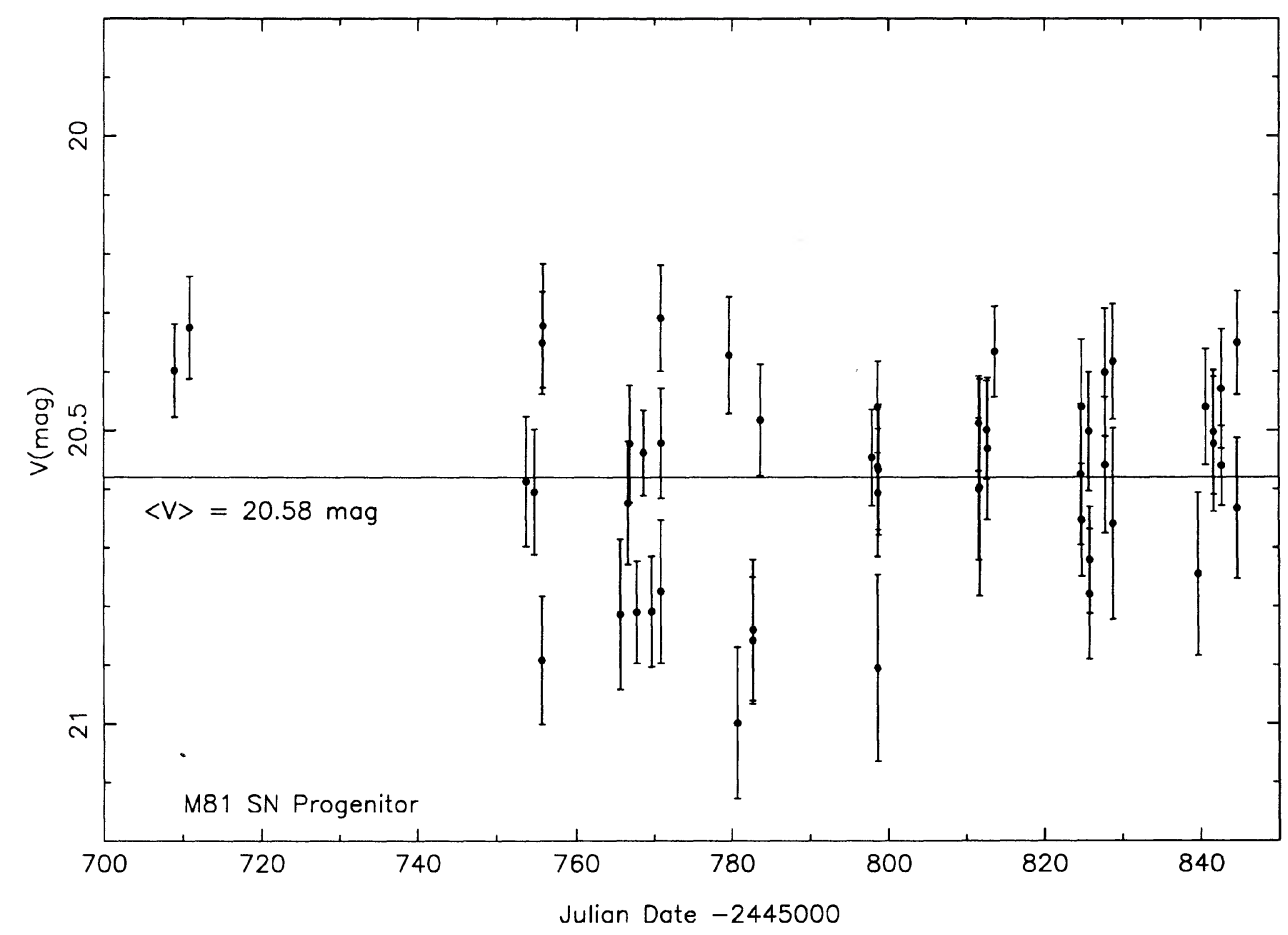

FIG. 1. The $V$ magnitudes for the progenitor of SN 1993J in M81 are plotted as a function of Julian Date for a period spanning 150 days in 1984.

typically 0.01 mag for the brightest local standards and 0.10 mag for the progenitor of the M81 SN. However, the predominant source of uncertainty is the spatial variations in the bright mottled background of the M81 arms and bulge, which in the vicinity of the M81 SN progenitor reaches $\approx 21.2 \mathrm{mag} / \operatorname{arcsec}^{2}$. Several different prescriptions for defining the appropriate local sky were tried during the course of reducing the data. Use of a small annulus 4 to 8 pixels in radius around the object seemed to give the most consistent results.

We find no evidence that the M81 SN progenitor is variable with an amplitude exceeding $0.2 \mathrm{mag}$, either from the size of the RMS deviations about the mean or from the lightcurve itself, which spans 150 days and is plotted in Fig. 1.

\section{COLORS OF THE PROGENITOR OF SN 1993J}

We use the results of Kent (1985) to transform our Thuann-Gunn $r$ magnitude into a Johnson $R$. This must then be transformed into a Kron-Cousins $R$ using the relationship of Landolt (1983). We obtain for Star A (the comparison object) $V-R=0.96 \pm 0.04 \mathrm{mag}$, while de Vaucouleurs et al. quote $V-R=0.86 \mathrm{mag}$, a comforting agreement. For the $\mathrm{SN}$ progenitor we obtain $\langle(V-R)\rangle=0.59 \pm 0.05 \mathrm{mag}$, where $R$ is the mean from two frames. (The difference between $R$ in the Kron-Cousins and in the Johnson system is insignificant.)

This is an excellent agreement with the values determined by Aldering et al. (1994) from the heterogeneous set of old CCD data, namely, $\langle V\rangle=20.60 \pm 0.16 \mathrm{mag}$ and $\langle(V-R)\rangle$ $=0.73 \pm 0.19 \mathrm{mag}$.

There is also good agreement with the mean $B-V$ color, where we obtain (from transforming $g-\langle V\rangle$ ) 1.20 \pm 0.05 , and Aldering et al. (1994) give as the mean of their heterogeneous data set $B-V=1.13 \pm 0.16 \mathrm{mag}$.

\section{COMMENTS ON VARIABILITY}

Our observations confirm and extend the earlier suggestion that the progenitor of SN 1993J in M81 is not variable at optical wavelengths, at least not with an amplitude of 0.2 mag or larger. This is also true of the progenitor of SN 1987A in the LMC (Hazen 1987).

With regard to stellar evolution, the last stages of stellar burning in the center of a massive star are so fast that the evolution of the envelope and of the core are decoupled. By the time carbon burning has begun in the core of a massive star, the outer layers have assumed their final configuration (Woosley \& Weaver 1986). Thus it is no surprise from this point of view that the luminosity emitted by the envelope of the supernova progenitor is observed to be constant $\approx 10 \mathrm{yr}$ prior to the explosion.

Although it is hard to predict what kind of binary system the progenitor star might have been in, the total light does not appear to have been dominated by the accretion disk itself, but rather by the supergiant SN progenitor star. Any binary system with a nondegenerate primary whose initial mass is at least 15 solar masses would have a large separation and a long period, at least a year. The light from the less massive and less evolved secondary could easily comprise less than $5 \%$ of the total emitted luminosity from the system, and hence be undetectable in our old and somewhat imprecise photometry. 


\section{COMMENTS ON THE PROGENITOR ITSELF}

We adopt from Freedman et al. (1994) a distance to M81 of $3.63 \mathrm{Mpc}$ (corresponding to a distance modulus of 27.80 mag) and a mean reddening of about $\mathrm{E}(B-V)=0.05$ mag. Thus $M_{V}$ for the M81 supernova progenitor is $\approx-7.4 \mathrm{mag}$, certainty typical of a supergiant. The $V, I$ (Kron-Cousins) color-magnitude diagram of the field of M81 from HST WFPC2 images is given in Hughes et al. (1994) while the older ground-based data for the brightest stars in M81 is given for several colors in Zickgraf \& Humphreys (1991). It is apparent that the progenitor star, with $V=20.58 \pm 0.17 \mathrm{mag}$ and $V-R=0.59 \pm 0.05 \mathrm{mag}$, is not a red supergiant in M81. Typical bright red supergiants in $\mathrm{M} 81$ have $V \approx 22$, with $V-I \approx 2$ mag and redder. For $(V-R) \approx(R-I)$, typical of most stars of that color (see Landolt 1983), we expect ( $V$ $-R) \approx 1.0 \mathrm{mag}$ for a typical red (M type) supergiant in M81.

The color temperature corresponding to $V-R=0.59$ $\pm 0.05 \mathrm{mag}$ is $6000 \pm 300 \mathrm{~K}$. The color temperature corresponding to the measured $B-R$ color is slightly lower, 4800 $\mathrm{K}$. We disregard the color temperature corresponding to $B-V$ since the wavelength leverage is so short and the errors in the magnitudes are relatively large. The consistency of these colors does not support the suggestion of Aldering et al. (1994) that substantial contamination of the light of the progenitor of SN 1993J occurs in the blue due to neighboring hotter stars. Thus the progenitor of the M81 supernova cor- responds to a late $\mathrm{G}$ supergiant, rather than an $\mathbf{M}$ supergiant.

\section{SUMMARY}

We have presented the 1984 data on the progenitor of SN 1993J in M81. These images are part of a longer series obtained as part of search for novae in nearby galaxies. By chance, two of the fields monitored in M81 include the location of SN 1993J. Based on 51 CCD frames taken in the $V$ filter, and several other taken through other filters, we find that the progenitor star is a late $G$ supergiant and it is not variable at the level of $0.2 \mathrm{mag}$ and over a time interval of 0.4 yr. Consistency with the compilation of previously published photometric observations of the progenitor star of SN 1993J by Aldering et al. (1994) extends the time scale over which variations were not detected, with very spotty coverage, to $\approx 10 \mathrm{yr}$.

J. G. C. is grateful to Alvio Renzini for helpful discussions and for the hospitality of the Institute of Astronomy at the University of Bologna. National Science Foundation (NSF) Grant No. AST-8212270 supported the search for novae in M81 and other nearby galaxies. J. K. D. was supported in part by a Student Undergraduate Research Fellowship from Caltech and in part by a REU supplement to NSF Grant No. AST-9213761.

\section{REFERENCES}

Aldering, G., Humphreys, R. M., \& Richmond, M. 1994, AJ, 107, 662 Arnett, W. D., Bahcall, J. N., Kirshner, R. P., \& Woosley, S. E. 1989, ARA\&A, 27, 629

Benson, P. J., et al. 1994, AJ, 107, 1453

Blakeslee, J., \& Tonry, J. 1993, IAUC, 5758

Cohen, J. G. 1993, IAUC, 5742

de Vaucouleurs, G., Corwin, Jr., H. G., \& Skiff, B. 1994, PASP, 106, 156

Filippenko, A. V., Matheson, T., \& Ho, L. C. 1993, ApJ, 415, L103

Freedman, W. L., \& Madore, B. F. 1988, ApJ, 332, L63

Freedman, W. L., et al. 1994, ApJ, 427, 628

Hazen, M. 1987, IAU Circ. No., 4365, 4367

Hoflich, P., Langer, N., \& Duschinger, M. 1993, A\&A, 275, L29

Hughes, S. M. G., et al. 1994, ApJ, 428, 143

Humphreys, R. M., Aldering, G. S., Bryja, C. O., \& Thurmes, P. M. 1993, IAUC, 5739

Kent, S. M. 1985, PASP, 97, 165

Landolt, A. 1983, AJ, 88, 439
Magnier, E., Lewin, W., Lubin, L., Zimmerman, U., \& Gabbiano, G. 1993, IAUC, 5741

Perelmutter, J. M. 1993, IAUC, 5736

Podsiadlowski, P., Hsu, J. J. L., Joss, P. C., \& Ross, R. R. 1993, Nature, 364, 509

Prugniel, P. 1993, IAUC, 5742

Richmond, M. W., Treffers, R. R., Filippenko, A. V., Paik, Y., Leibundgut, B., Schulman, E., \& Cox, C. V. 1994, AJ, 107, 1022

Ripero, J. 1993, IAUC, 5731

Salzer, J. J., Herbst, W. E., \& Vinton, G. 1993, IAUC, 5741

Schmidt, B. P., et al. 1993, Nature, 364, 600

Swartz, D. A., Clocchiatti, A., Benjamin, R., Lester, D. F., \& Wheeler, J. C. 1993, Nature, 365, 232

Thuan, T. X., \& Gunn, J. E. 1976, PASP, 88, 543

Tomaney, A., \& Crotts, A. 1993, IAUC, 5737

Woosley, S. E., \& Weaver, T. A. 1986, ARA\&A, 24, 205

Zickgraf, F.-J., \& Humphreys, R. M. 1991, AJ, 102, 113 